\title{
Average circular D-distance and circular D-Wiener index of K-regular graphs
}

\author{
Janagam Veeranjaneyulu ${ }^{a}$, Peruri Lakshmi Narayana Varma ${ }^{b}$ \\ $\mathbf{a}, \mathbf{b}$ \\ Division of Mathematics, Department of S \& H, Vignan's Foundation For \\ Science, Technology \& Research, Vadlamudi - 522213, Guntur, India
}

Article History: Received: 11 January 2021; Accepted: 27 February 2021; Published online: 5 April 2021

\begin{abstract}
The circular -distance between nodes of a graph is obtained by the sum of detour -distance and -distance. The average circular -distance between the nodes of a graph is the sum of average of the detour -distance and -distances. In this paper, we deal with the average circular -distance between nodes of graph. We compute the relation between circular Wiener index and circular -Wiener index of -regular graphs. We obtained results on circular -Wiener index of some special graphs
\end{abstract}

Keywords: Average circular -distance, detour Wiener index, detour -Wiener index, circular -Wiener index Mathematics Subject Classification: 05C12.

\section{Introduction}

The idea of distance is one of the significant idea in investigation of graphs. It is used to test the isomorphism of graphs, connectivity problems and convexity of graphs etc.

In addition to the geodesic distance, $d(r, s)$, we have detour distance (introduced by Chartrand et al in [3], average distance and dominating number (introduced by Dankelmann in [4,5] ), mean distance in graphs (introduced by Doyale in [6] ), superior distance (introduced by Kathiresan and Marimuthu in [7] ), signal distance (introduced by Kathiresan and Marimuthu in [8] ), average $D$-distance (introduced by Reddy babu, Varma in [11] ), average detour $D$-distance (introduced by Venkateswara Rao, Varma in [12] ).

In previous article (in [15]), the authors presented the idea of circular $D$-distance in graphs by adding detour $D$-distance and $D$-distance. A relation between Wiener index for $k$-regular graphs and $D$-index (was obtained by Ahmed Mohammed Ali and Asmaa Salah Aziz in [1]).

In this article we introduce, the concept of average circular $D$-distance between nodes of a graph and study some of its properties. Further we compute the average circular $D$-distance of some classes of graphs. We introduce the concept of Wiener detour $D$-index of a graph $H$. We obtain a relation between detour Wiener index and detour $D$-Wiener index of any regular graph $H$. Further we compute detour $D$-Wiener index of some special graphs. Finally we obtain a relation between circular Wiener index and circular $D$-Wiener index of regular graph $H$. Furthermore, this distance is also used in molecular dynamics of physics, crystallography, lattice statistics and physics.

Throughout this article we consider connected and simple graphs. For any unexplained terminology and symbols, we refer the book [2].

\section{Average circular $D$-distance}

In this section we given some definitions for later use.

Definition 2.1 Let $H$ be a connected graph of order $n$, the average distance (respectively, average detour distance) between nodes of $H$, denoted by $\mu(H)$ (respectively, $\mu_{D}(H)$ ), is defined as $\mu(H)=\frac{1}{2}\left(\begin{array}{l}n \\ 2\end{array}\right)^{-1} \sum_{r, s \in V(H)} d(r, s) \quad \mu_{D}(H)=\frac{1}{2}\left(\begin{array}{l}n \\ 2\end{array}\right)^{-1} \sum_{r, s \in V(H)} D(r, s)$ ) where $d(r, s)$ and $D(r, s)$ denote the distance and detour distance between the nodes of $r$ and $s$ respectively.

Definition 2.2. Let $H$ be a connected graph of order $n$, the average $D$ - distance (respectively, average detour $D-$ distance) between nodes of $H$, denoted by $\mu^{D}(H)$ (respectively, $\mu_{D}^{D}(H)$ ), is defined as $\mu^{D}(H)=\frac{1}{2}\left(\begin{array}{l}n \\ 2\end{array}\right)^{-1} \sum_{r, s \in V(H)} d^{D}(r, s) \mu_{D}^{D}(H)=\frac{1}{2}\left(\begin{array}{l}n \\ 2\end{array}\right)^{-1} \sum_{r, s \in V(H)} D^{D}(r, s) \quad$ ) where $d^{D}(r, s)$ and $D^{D}(r, s)$ denote the $D$ - distance and detour $D$ - distance between the nodes of $r$ and $s$ respectively. 
Definition 2.3 [16]. In a graph $H, r, s$ are any two nodes and circular distance between them is defined as $\operatorname{cir}(r, s)=\left\{\begin{array}{cc}0 & \text { if } r=s \\ d(r, s)+D(r, s) & \text { if } r \neq s\end{array}\right.$

Definition 2.4 [15]. In a graph $H, r, s$ are any two nodes and circular $D$-distance between them is defined as

$$
\operatorname{cir}^{D}(r, s)=\left\{\begin{array}{cc}
0 & \text { if } r=s \\
\min \left\{l^{D}(S)\right\}+\max \left\{l^{D}(S)\right\} & \text { if } r \neq s
\end{array}\right.
$$

Now, average circular $D$ - distance of a graph as shown below.

Definition 2.5. $\mu_{C}^{D}(H)$, call it as average circular $D$-distance $(A V C D D)$ between nodes of $H$, where $H$ is a connected graph of order $n$, defined as sum of average detour $D$ - distance and average $D$ - distance, i.e., $\mu_{C}^{D}(H)=\frac{1}{2}\left(\begin{array}{l}n \\ 2\end{array}\right)^{-1} \sum_{r, s \in V(H)} \operatorname{cir}^{D}(r, s)$ and $s$. where $\operatorname{cir}^{D}(r, s)$ denotes the circular $D-$ distance between the nodes $r$

Definition 2.6. Let $H$ be a connected graph of order $n$ with node set $\left\{r_{1}, r_{2}, r_{3}, \cdots, r_{n}\right\}$ and having $m$ edges. The circular $D$ - distance matrix of $H$, represented as, $D_{C}^{D}(H)$, is defined as $D_{C}^{D}(H)=\left[\operatorname{cir}_{i, j}^{D}\right]_{n \times n}$ where $\operatorname{cir}_{i, j}^{D}=\operatorname{cir}^{D}\left(r_{i}, r_{j}\right)$ is the circular $D$ - distance of the nodes $r_{i}$ and $r_{j}$

Definition 2.7. The total circular $D$ - distance (TCDD) of graph $H$ is the number given by ${ }^{r, s}$

\section{Results on average circular $D$-distance}

Now we prove some results on $A V C D D$ between nodes. We begin with theorem which connects the number of nodes and $A V C D D$. This leads to some more results.

Theorem 3.1. Let $H_{1}$ and $H_{2}$ be two graphs having equal number of nodes and $d_{C}^{D}\left(H_{1}\right)<d_{C}^{D}\left(H_{2}\right)$. If $\left|E_{1}\right|<\left|E_{2}\right|_{\text {then }} \mu_{C}^{D}\left(H_{1}\right)<\mu_{C}^{D}\left(H_{2}\right)$.

Proof: Since $d_{C}^{D}\left(H_{1}\right)<d_{C}^{D}\left(H_{2}\right)$, the biggest entry in the circular $D$ - distance matrix of $H_{1}<H_{2}$ and this leads total circular $D-$ distance value to increase. Since orders are same and $\left|E_{1}\right|<\left|E_{2}\right|$. Hence $\mu_{C}^{D}\left(H_{1}\right)<\mu_{C}^{D}\left(H_{2}\right)$.

Corollary 3.2 Let ${ }^{H_{1}}$ and ${ }^{H_{2}}$ contains equal number of nodes and $d_{C}^{D}\left(H_{1}\right)=d_{C}^{D}\left(H_{2}\right)$. If $\left|E_{1}\right|=\left|E_{2}\right|$ then $\mu_{C}^{D}\left(H_{1}\right)=\mu_{C}^{D}\left(H_{2}\right)$

Theorem 3.3. Let $H_{1}$ and $H_{2}$ be two graphs having equal number of nodes and $d_{C}^{D}\left(H_{1}\right)<d_{C}^{D}\left(H_{2}\right)$. If $\delta\left(H_{1}\right)<\delta\left(H_{2}\right)$ then $\mu_{C}^{D}\left(H_{1}\right)<\mu_{C}^{D}\left(H_{2}\right)$.

Proof: Let $H_{1}$ and $H_{2}$ be two connected graphs having equal number of modes and $d_{C}^{D}\left(H_{1}\right)<d_{C}^{D}\left(H_{2}\right)$. Then clearly $\delta\left(H_{1}\right)<\delta\left(H_{2}\right) \Rightarrow\left|E_{1}\right|<\left|E_{2}\right|$. Then from theorem 3.1 we get $\mu_{C}^{D}\left(H_{1}\right)<\mu_{C}^{D}\left(H_{2}\right)$.

Corollary 3.4 Let $H_{1}$ and $H_{2}$ contains equal number of nodes and $d_{C}^{D}\left(H_{1}\right)=d_{C}^{D}\left(H_{2}\right) \cdot{ }_{\text {If }} \delta\left(H_{1}\right)=\delta\left(H_{2}\right)$ then $\mu_{C}^{D}\left(H_{1}\right)=\mu_{C}^{D}\left(H_{2}\right)$.

Theorem 3.5. Let ${ }^{H_{1}}$ be a spanning subgraph of $H_{2}$ then $\mu_{C}^{D}\left(H_{1}\right)<\mu_{C}^{D}\left(H_{2}\right)$. 
Proof: Let $H_{1}$ be a spanning subgraph of $H_{2}$. Then the number of nodes of $H_{1}$ and $H_{2}$ are same and number of edges of $H_{1}$ is less than that of $H_{2}$. Then by theorem 3.1 we get $\mu_{C}^{D}\left(H_{1}\right)<\mu_{C}^{D}\left(H_{2}\right)$.

\section{Results on some families of graphs}

Here we compute the $A V C D D$ for some families of graphs.

Theorem 4.1. The AVCDD of the complete graph $K_{m}$ is $m^{2}+2 m-2$.

Proof: $\quad$ We know that, $K_{m}$ is a $m-1$ regular graph and the circular $D$ - distance between each pair of nodes is $m^{2}+2 m-2$ (see theorem 4.1 in [14]). Thus the TCDD is $2\left(\begin{array}{l}n \\ 2\end{array}\right)\left(m^{2}+2 m-2\right)$. Hence $\mu_{C}^{D}\left(K_{m}\right)=\frac{1}{2}\left[\frac{T C D D}{\left(\begin{array}{l}n \\ 2\end{array}\right)}\right]=m^{2}+2 m-2$

Remark 4.2. In a complete graph $K_{m}, \mu_{C}^{D}\left(K_{m}\right)=d_{C}^{D}\left(K_{m}\right)=d_{C}^{D}\left(K_{m}\right)$.

Theorem 4.3. The AVCDD of the cycle graph $C_{m}$ is $3 m+4$.

Proof: $\quad$ Each node of the cyclic graph $C_{m}$ has 2 adjacent nodes. The circular $D$ - distance between each pair of nodes is $3 m+4$ (see theorem 4.3 in [14]). Thus the TCDD is $2\left(\begin{array}{l}n \\ 2\end{array}\right)(3 m+4)$. Hence $\mu_{C}^{D}\left(C_{m}\right)=\frac{1}{2}\left[\frac{T C D D}{\left(\begin{array}{l}n \\ 2\end{array}\right)}\right]=3 m+4$

Remark 4.4: In a cycle graph $C_{m}, \mu_{C}^{D}\left(C_{m}\right)=d_{C}^{D}\left(C_{m}\right)=d_{C}^{D}\left(C_{m}\right)$.

Theorem 4.5: The AVCDD of a path graph $P_{n}$ is $\frac{4 a_{n}}{n}$, where $a_{n}=a_{n-1}+n+1$ with $a_{1}=0$.

Proof: Consider the circular $D$ - distance matrix of the path graph $P_{n}$, which is $n \times n$ symmetric matrix.

$$
\left[\begin{array}{ccccccc}
0 & 8 & 14 & 10 & \cdots & 6 n-10 & 6 n-4 \\
& 0 & 10 & 16 & \cdots & 6 n-14 & 6 n-8 \\
& & 0 & 10 & \cdots & 6 n-24 & 6 n-18 \\
& & & \vdots & \cdots & \vdots & \vdots \\
& & & & 0 & 10 & 14 \\
& & & & & 0 & 8 \\
& & & & & & 0
\end{array}\right]
$$

Summation of all elements in this right triangular matrix, we have the TCDD is $4 a_{n}(n-1)$, where $a_{n}$ is a constant given by $\{3,7,12,18,25, \cdots\}$ or recursively $a_{n}=a_{n-1}+n+1$ with $a_{1}=0$. Hence the total circular $D-$ distance is $4 a_{n}(n-1)$. 
Thus AVCDD of $P_{n}$ is

$$
\mu_{C}^{D}\left(C_{m}\right)=\frac{1}{2}\left[\frac{T C D D}{\left(\begin{array}{l}
n \\
2
\end{array}\right)}\right]=\left[\frac{4 a_{n}(n-1)}{\frac{1}{2} n(n-1)}\right]=\frac{4 a_{n}}{n}
$$

Theorem 4.6. The AVCDD of star graph ${ }^{t_{n, 1}}$ is $\frac{2 n(n+5)}{n-1}$.

Proof: In a star graph ${ }^{S t_{n, 1}}$, let $v_{0}$ be the node which is adjacent to all other nodes. The circular $D$ - distance between any two nodes is either $2 n+4$ or $2 n+8$ (see theorem 3.14 in [15]). Finally the total circular $D-$ distance is $2 n(2 n+4)+2\left(\begin{array}{l}n \\ 2\end{array}\right)(2 n+8)$. Thus AVCDD of $S t_{n, 1}$ is

$$
\begin{aligned}
\mu_{C}^{D}\left(S t_{n, 1}\right) & =\frac{1}{2}\left[\frac{T C D D}{\left.\left(\begin{array}{l}
n \\
2
\end{array}\right)\right]}\right. \\
& =\frac{2(2 n+4)+(n-1)(2 n+8)}{n-1} \\
& =\frac{2 n(n+5)}{n-1}
\end{aligned}
$$

\section{Theorem 4.7.}

1. The AVCDD of complete bipartite graph $K_{\mathrm{x}, y}$ is

$$
\frac{(x+1) y^{3}+\left(3 x^{2}+6 x+1\right) y^{2}+\left(3 x^{3}+6 x^{2}-7 x-2\right) y+\left(x^{4}+x^{3}-2 x^{2}\right)}{y^{2}+(2 x-1) y+x^{2}-x}
$$

2. The AVCDD of complete bipartite graph $K_{\mathrm{x}, x}$ is $\frac{4 x^{3}+6 x^{2}-4 x}{2 x-1}$

Proof: (1) The node set of $K_{\mathrm{x}, y}$ can be split as $U_{1} \cup U_{2}$, where $U_{1}=\left\{v_{1}, v_{2}, v_{3}, \cdots v_{x}\right\}$, $U_{2}=\left\{w_{1}, w_{2}, w_{3}, \cdots w_{y}\right\} . \quad$ Then $\quad \operatorname{cir}^{D}\left(v_{i}, v_{j}\right)=x(x+5)+y(x+1)+2, \operatorname{cir}^{D}\left(w_{i}, w_{j}\right)=x^{2}+x y+2(x+y)$, $\operatorname{cir}^{D}\left(v_{i}, w_{j}\right)=x(x+3)+y(x+1)$. Thus the total circular $D-$ distance is $\frac{(x+1) y^{3}+\left(3 x^{2}+6 x+1\right) y^{2}+\left(3 x^{3}+6 x^{2}-7 x-2\right) y+\left(x^{4}+x^{3}-2 x^{2}\right)}{y^{2}+(2 x-1) y+x^{2}-x}$. $\mu_{C}^{D}\left(K_{x, y}\right)=\frac{1}{2}\left[\frac{T C D D}{x+y}{ }_{2}\right]$

Hence the average circular $D$ - distance

$$
=\frac{(x+1) y^{3}+\left(3 x^{2}+6 x+1\right) y^{2}+\left(3 x^{3}+6 x^{2}-7 x-2\right) y+\left(x^{4}+x^{3}-2 x^{2}\right)}{y^{2}+(2 x-1) y+x^{2}-x}
$$

(2) The node set of $K_{\mathrm{x}, x}$ can be split as $U_{1} \cup U_{2}$, where $U_{1}=\left\{v_{1}, v_{2}, v_{3}, \cdots v_{x}\right\}, U_{2}=\left\{w_{1}, w_{2}, w_{3}, \cdots w_{x}\right\}$. Then $\operatorname{cir}^{D}\left(v_{i}, v_{j}\right)=2 x^{2}+4 x, \operatorname{cir}^{D}\left(w_{i}, w_{j}\right)=2 x^{2}+4 x, \operatorname{cir}^{D}\left(v_{i}, w_{j}\right)=2 x^{2}+4 x$. 
distance is

$$
2\left[\left(\begin{array}{l}
x \\
2
\end{array}\right)\left(2 x^{2}+4 x\right)+x^{2}\left(2 x^{2}+4 x\right)+\left(\begin{array}{l}
x \\
2
\end{array}\right)\left(2 x^{2}+4 x\right)\right]=2\left[\left(2 x^{2}-x\right)\left(2 x^{2}+4 x\right)\right] .
$$

Hence the average

circular $D$ - distance is

$$
\mu_{C}^{D}\left(K_{x, x}\right)=\frac{1}{2}\left[\frac{T C D D}{2 x}+=\frac{4 x^{3}+6 x^{2}-4 x}{2 x-1}\right.
$$

\section{The index of detour $D$-distance for connected $\boldsymbol{k}$-regular graphs}

Here we begin with some definitions on Wiener index and detour $D$ - Wiener index.

Definition 5.1. Let $H$ be a graph of order $n$, the Wiener index (respectively, detour Wiener index) between nodes of $H$, denoted by $W(H)$ (respectively, $D(H)$ ), is defined as $W(H)=\frac{1}{2} \sum_{\forall r, s \in V(H)} d(r, s)$ $D(H)=\frac{1}{2} \sum_{\forall r, s \in V(H)} D(r, s)$ nodes of $r$ and $s$ respectively.

Definition 5.2. The total $D-$ distance or $D-$ index of a graph $H$ is defined as $W^{D}(H)=\frac{1}{2} \sum_{\forall r, s \in V(H)} d^{D}(r, s)$.

Definition 5.3. The total detour $D$ - distance or detour $D$-index of a graph $H$ is defined as $W_{D}^{D}(H)=\frac{1}{2} \sum_{\forall r, s \in V(H)} D^{D}(r, s)$

Now we prove a relation between detour Wiener index and detour $D-$ Wiener index of $k-$ regular graphs.

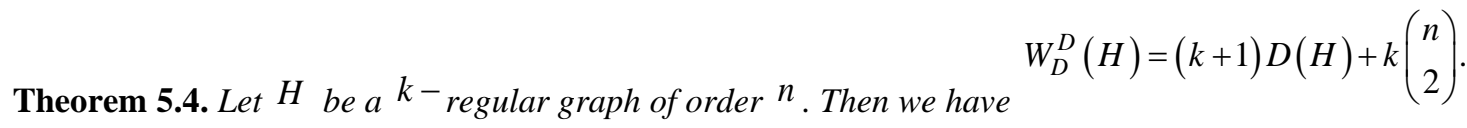

Proof: We have, by definition

$$
\begin{aligned}
& W_{D}^{D}(H)=\frac{1}{2} \sum_{\forall r, s \in V(H)} D^{D}(r, s) \\
& =\frac{1}{2} \sum_{\forall r, s \in V(H)}\left[\max _{p}\left\{l(P)+\sum_{w \in V(P)} \operatorname{deg}(w)\right\}\right] \\
& =\frac{1}{2} \sum_{\forall r, s \in V(H)}\left\{D(r, s)+\sum_{w \in V(P: r-s \text { detour path })} \operatorname{deg}(w)\right\} \\
& =\frac{1}{2} \sum_{\forall r, s \in V(H)} D(r, s)+\frac{1}{2} \sum_{\forall r, s \in V(H)}\left\{\sum_{w \in V(P: r-s \text { detour path })} \operatorname{deg}(w)\right\} \\
& =D(H)+\frac{1}{2} \sum_{\forall r, s \in V(H)}\left\{\sum_{w \in V(P: r-s \text { detour path })} \operatorname{deg}(w)\right\}
\end{aligned}
$$

Since $H$ is $k$-regular then $\operatorname{deg}(w)=k$, for all $w \in V(H)$ and between $r$ and $s$ every detour path contains $D(r, s)+1$ nodes with $\operatorname{deg}(w)=k$ for all $w$. Thus 


$$
\begin{aligned}
W_{D}^{D}(H) & =D(H)+\frac{1}{2} \sum_{\forall r, s \in V(H)}(D(r, s)+1) k \\
& =D(H)+\frac{1}{2} \sum_{\forall r, s \in V(H)} D(r, s) k+\frac{k}{2} \sum_{\forall r, s \in V(H)} 1 \\
& =D(H)+k D(H)+k\left(\begin{array}{l}
n \\
2
\end{array}\right) \\
& =D(H)(k+1)+k\left(\begin{array}{l}
n \\
2
\end{array}\right)
\end{aligned}
$$

Hence

$W_{D}^{D}(H)=(k+1) D(H)+k\left(\begin{array}{l}n \\ 2\end{array}\right)$.

(2) For the complete graph $K_{n}, W_{D}^{D}\left(K_{n}\right)=\left(n^{2}-1\right)\left(\begin{array}{l}n \\ 2\end{array}\right)$.

Proof. This proof follows from the theorem 1 and corollary 1 of [1].

Theorem 5.6. For the complete bipartite graph $K_{x, x}, W_{D}^{D}\left(K_{x, x}\right)=4 x^{4}+x^{3}-4 x^{2}+2 x$.

Proof: $\quad$ The proof is similar to that of 6.5 below.

\section{The detour $D$-index of some special graphs}

Now we find the detour $D$-index of graphs which are not regular.

Now we begin with path graph.

Theorem 6.1. The detour $D-$ index of a path graph $P_{n}$ is $\frac{1}{2} n^{3}+n^{2}-\frac{7}{2} n+2$.

Proof. In a path graph $P_{n}$, both $D$ - distance and detour $D$ - distance are equal. Thus see the proof of theorem 4 of [1].

Theorem 6.2. The detour $D-$ index of a star graph $S t_{n, 1}$ is $\frac{1}{2} n^{2}(n+5)$.

Proof. In a star graph $S t_{n, 1}$ both $D$-distance and detour $D$ - distance are equal. Thus see the proof of theorem 4 of [1].

Theorem 6.3. The detour $D-$ index of a friendship graph $F_{n}$ is $\frac{1}{2}\left[n^{3}+7 n^{2}-17 n+9\right]$.

Proof. Let $F_{n}$ be a friendship graph whose nodes can be listed as $V\left(F_{n}\right)=\left\{r_{1}, r_{2}, r_{3}, \cdots r_{n}\right\}$, where $\operatorname{deg}\left(r_{1}\right)=n-1$ and $\operatorname{deg}\left(v_{i}\right)=2$, for all $i=2,3, \cdots n$. Then we have three cases:

Case 1: If $r_{1} r_{i} \in E\left(F_{n}\right)$ then $D^{D}\left(r_{1}, r_{i}\right)=n+5$, for $i=2,3, \cdots, n$.

Case 2: If $r_{2 i} r_{2 i+1} \in E\left(F_{n}\right)$ then $D^{D}\left(r_{2 i}, r_{2 i+1}\right)=n+5$, for $i=2,3, \cdots, \frac{n-1}{2}$ and number of such pairs of $\left(r_{2 i}, r_{2 i+1}\right)_{\text {is }} \frac{n-1}{2}$ 
Case 3: If $r_{2 i-1} r_{2 i} \notin E\left(F_{n}\right)$ then the path $P$ consists from $r_{2 i-1}, r_{i}, r_{1}, r_{2 i}$. Hence $D^{D}\left(r_{2 i}, r_{2 i-1}\right)=n+8$, for $i=2,3, \cdots, \frac{n-1}{2}$. The number of such pairs are $\frac{(n-1)(n-3)}{2}$ $W_{D}^{D}\left(F_{n}\right)=(n-1)(n+5)+\frac{1}{2}(n+5)(n-1)+\frac{1}{2}(n+8)(n-1)(n-3)=\frac{1}{2}\left[n^{3}+7 n^{2}-17 n+9\right]$.

Theorem 6.4. For a wheel graph $W_{1, n}$, we have $W_{D}^{D}\left(W_{1, n}\right)=\frac{1}{2}\left(5 n^{2}(n+1)\right)$ and for $n \geq 3$.

Proof: Let $\left\{r_{0}, r_{1}, \ldots, r_{n}\right\}$ be the node set of wheel graph. Suppose that $r_{0}$ is adjacent to all other nodes. Thus $\operatorname{deg}\left(r_{0}\right)=n_{\text {\$and }} \operatorname{deg}\left(r_{i}\right)=3$, for $i=1,2, \cdots, n$. Hence $D^{D}\left(r_{i}, r_{j}\right)=5 n$ for all $i, j$ (theorem 4.5 of [12]). The number of this type of pairs is $\left(\begin{array}{c}n+1 \\ 2\end{array}\right)$. Thus total detour $D$ - distance is $2\left(\begin{array}{c}n+1 \\ 2\end{array}\right)(5 n)$. $W_{D}^{D}\left(W_{1, n}\right)=\frac{1}{2}\left(5 n^{2}(n+1)\right)$.

Now, we look at complete bipartite graph.

Theorem 6.5. For a complete bipartite graph $K_{x, y}$, having ${ }^{x+y}$ nodes with ${ }^{x<y}$. Then we have $W_{D}^{D}\left(K_{x, y}\right)=\frac{1}{2}\left[x y^{3}+\left(3 x^{2}+2 x\right) y^{2}+\left(3 x^{3}+2 x^{2}-5 x\right) y+\left(x^{4}-3 x^{2}+2 x\right)\right]$

Proof. In $K_{x, y}$, the node set can be divided into sets $U_{1}$ and $U_{2}$. The number of nodes of $U_{1}$ and $U_{2}$ is ${ }^{x}$ and ${ }^{y}$ respectively.

Now, see the three cases:

Case1: If $r, r^{\prime} \in U_{1}$ then $D^{D}\left(r, r^{\prime}\right)=x^{2}+x y+x-2$ (theorem 4.6 of [12]), this is true for all $\frac{x(x-1)}{2}$ pairs of nodes.

Case2: $r, r^{\prime} \in U_{2}$ then $D^{D}\left(r, r^{\prime}\right)=x^{2}+x y+3 x$ (theorem 4.6 of $\backslash$ cite $\left.\{\mathrm{VV}\}\right)$, this is correct for all $\frac{y(y-1)}{2}$ couple of nodes.

$$
\begin{aligned}
& \text { Case 3: If } r \in U_{1} \text { and } r^{\prime} \in U_{2} \text { then } D^{D}\left(r, r^{\prime}\right)=x^{2}+2 x+x y-1 \text { (theorem } 4.6 \text { of [12]), this is correct for } \\
& \text { all couple of nodes. The total detour } D-\text { distance is } \\
& 2\left[\left(\begin{array}{l}
x \\
2
\end{array}\right)\left(x^{2}+x y+x-2\right)+x y\left(x^{2}+2 x+x y-1\right)+\left(\begin{array}{l}
y \\
2
\end{array}\right)\left(x^{2}+x y+3 x\right)\right] . \\
& =\frac{1}{2}\left[x y^{3}+\left(3 x^{2}+2 x\right) y^{2}+\left(3 x^{3}+2 x^{2}-5 x\right) y+\left(x^{4}-3 x^{2}+2 x\right)\right] .
\end{aligned}
$$

\section{Circular $D$-Wiener index of $k$-regular graphs}

Here we introduce the definition of circular \$D\$-Wiener index and compute the connection between circular $D$ - Wiener index of $k$ - regular graphs and circular Wiener index.

Definition 7.1. The circular $D$ - Wiener index (respectively, circular Wiener index) between nodes of $H$, denoted by $W_{C}^{D}(H)$ (respectively, $W^{C}(H)$ ), is defined as

$$
W_{C}^{D}(H)=\frac{1}{2} \sum_{\forall r, s \in V(H)} \operatorname{cir}^{D}(r, s)
$$

(respectively, 
$W^{C}(H)=\frac{1}{2} \sum_{\forall r, s \in V(H)} \operatorname{cir}(r, s)$

), where $\operatorname{cir}^{D}(r, s)$ and $\operatorname{cir}(r, s)$ denotes the circular $D$ - distance (see in [15]) and circular distance (see in [16]) between the nodes of $r$ and $s$ respectively.

Now we compute the relation between circular $D$ - Wiener index and circular Wiener index.

Theorem 7.2. Let $H$ be a $k$ - regular graph of order $n$. Then we have $W_{C}^{D}(H)=$ $(k+1) W^{C}(H)+k n(n-1)$

Proof. The index of $D$ - distance for a $k$-regular graph of $H$ is $W^{D}(H)=(k+1) W(H)+k\left(\begin{array}{l}n \\ 2\end{array}\right)$ (see theorem 3 in [1] ). The detour $D$ - Wiener index for a connected $k$ - regular graph is $W_{D}^{D}(H)=(k+1) D(H)+k\left(\begin{array}{l}n \\ 2\end{array}\right)$ (see theorem 5.4). Hence the next proof follows from the definition circular $D$ Wiener index. Hence $W_{C}^{D}(H)=(k+1) W^{C}(H)+k n(n-1)$.

Theorem 7.3. For the complete graph $K_{m}, \quad W_{C}^{D}\left(K_{m}\right)=\left(m^{2}+2 m-2\right)\left(\begin{array}{l}m \\ 2\end{array}\right)$

Proof; See the proof of theorem 4.1

Theorem 7.4. The circular $D$ - Wiener index of a cycle graph $C_{m}$ is $(3 m+4)\left(\begin{array}{l}m \\ 2\end{array}\right)$.

Proof; See the proof of theorem 4.3

Theorem 7.5. The circular D-Wiener index of a complete bipartite graph $K_{x, x}$ is $4 x^{4}+6 x^{3}-4 x^{2}$.

Proof; See the proof of theorem 4.7

Theorem 7.6. The circular $D$ - Wiener index of a path graph $P_{n}$ is $2 a_{n}(n-1)$.

Proof; See the proof of theorem 4.5

Theorem 7.7. The circular $D-$ Wiener index of a star graph $S t_{n, 1}$ is $n^{3}+5 n^{2}$.

Proof; See the proof of theorem 4.6

Theorem 7.8. The circular D- Wiener index of a complete bipartite graph $K_{x, y}$ is $\frac{1}{2}\left[(x+1) y^{3}+\left(3 x^{2}+6 x+1\right) y^{2}+\left(3 x^{3}+6 x^{2}-7 x-2\right) y+\left(x^{4}+x^{3}-2 x^{2}\right)\right]$.

Proof; See the proof of theorem 4.7

\section{Conclusion}

In this paper, we discuss properties of $A V C D D$ and we find the average circular $D$ - distance of some families of graphs. Further, we find a formula giving a connection between the detour Wiener index and detour $D-$ Wiener index. We find the connection between circular Wiener index and circular $D-$ Wiener index of $k-$ regular graphs.

In future work, we find average circular $D$ - distance between arcs of a graph and we can find the relation between circular $D$ - Wiener index and average circular $D$ - distance between arcs of a graph.

\section{References}

A. M. Ali, A. S. Aziz, A relation between D-index and Wiener index for $r$-regular graphs, International Journal of Mathematics and Mathematical Sciences. 2020 (2020), 1-6.

1. F. Buckley, F. Harary, Distance in Graph, Addison-Wesley, Redwood City, 1990, 215--240.

2. G. Chartrand, H. Escuadro, P. Zhang, Detour distance in graphs, J. Combin. Comput. 53 (2005), 75-94. 
3. P. Dankelmann, Average distance and dominating number, Discrete Applied Mathematics. (80) (1997), 21-35.

4. P. Dankelmann, S. Mukwembu, H. C. Swart, Average distance and edge connectivity. I*, Siam J. Discrete Math (22) (2008), 92-101.

5. J. K. Doyale, J. E. Graver, Mean distance in a graphs, Discrete Mathematics. (17) (1977), 147-154.

6. K. M. Katheresan, G. Marimuthu, Superior distance in graphs, J. Combin. comput. (61) (2007), 73-80.

7. K. M. Kathiresan, R. Sumathi, A study on signal distance in graphs, Algebra, Graph Theory and their applications. Narosa publishing house Pvt. Ltd, (2010), 50-54.

8. K. M. Kathiresan, C. Parameswaran, G. Marimuthu and S. Arockiaraj, Detour Wiener indices of graphs, Bulletin of the ICA ( 62) (2011), 33-47.

9. Reddy Babu,D., Varma, P. L. N., D-distance in graphs, Gold. Res. Thoughts, 2 (2013) 53-58.

10. D. Reddy Babu, P. L. N Varma, Average D-distance between vertices of a graph, Italian Journal of Pure and Applied Mathematics. (33) (2014), 293-298.

11. V. Venkateswara Rao, P. L. N Varma, Detour distance in graphs W. R. T D-distance, Ponte International Journal of Science and Research. (73) (2017), no. 7, 19-28.

12. V. Venkateswara Rao, P. L. N Varma, Average detour D-distance between vertices of a graph, Advances in Mathematics: Scientific Journal. (9) (2020), no.7, 4721-4727. https://doi.org/10.37418/amsj.9.7.38.

13. J. Veeranjaneyulu, P. L. N Varma, Estimating circular distance in graph nodes, International Journal of Engineering and Advanced Technology (IJEAT). (9) (2019), no. 1S5, 311-316.

14. J. Veeranjaneyulu, P. L. N Varma, Circular D-distance and path graphs, International Journal of Recent Technology and Engineering (IJRTE)\}. (7) (2019), no. 5S4, 219-223.

15. P. L.N. Varma, J. Veeranjaneyulu, Study of circular distance in graphs. Preprint, (2021). 